\title{
RENDIMIENTO ESCOLAR Y AUTOCONCEPTO EN EDUCACIÓN PRIMARIA. RELACIÓN Y ANÁLISIS POR GÉNERO
}

\author{
Lucía Herrera Torres \\ Departamento de Psicología Evolutiva y de la Educación, Universidad de Granada, Melilla, España \\ Mohamed Al-Lal Mohand \\ CEIP Pedro de Estopiñán, Melilla, España \\ Laila Mohamed Mohand \\ CEIP Pedro de Estopiñán, Melilla, España \\ luciaht@ugr.es, mam.melilla@gmail.com, lafu@ugr.es \\ https://doi.org/10.17060/ijodaep.2017.n1.v3.1000
}

Fecha de Recepción: 23 Marzo 2017

Fecha de Admisión: 1 Abril 2017

\section{RESUMEN}

Este trabajo pretende, por una parte, analizar la relación entre autoconcepto y rendimiento escolar. Por otra, establecer si existen diferencias en ambos constructos en función del género de los estudiantes. En el estudio participaron 422 estudiantes de Educación Primaria, de los cuales 198 eran niños y 224 niñas. El $48.3 \%$ se encontraba en quinto curso y el $51.7 \%$ en sexto curso. Los niños estudiaban en ocho de los doce centros públicos de educación infantil y primaria de la ciudad autónoma de Melilla. Como instrumentos de recogida de información se emplearon dos. En primer lugar, un registro de las calificaciones finales en las asignaturas de Conocimiento del Medio, Lengua Castellana y Matemáticas de cada estudiante, facilitado por los tutores de los alumnos. En segundo lugar, el Cuestionario de Autoconcepto Forma 5 (AF-5). Los resultados pusieron de manifiesto correlaciones positivas entre las calificaciones de las tres asignaturas evaluadas y tres de las cinco dimensiones del autoconcepto: académico, social y familiar. En lo relativo al análisis en función del género, se encontraron en las calificaciones para la asignatura de Lengua Castellana, de modo que las niñas puntuaron más alto que los niños. Además, también se encontraron diferencias por género en las dimensiones del autoconcepto académico, emocional y familiar. Así, las niñas presentaban un autoconcepto académico y familiar superior a los niños, mientras que en el autoconcepto emocional fueron los niños los que puntuaron más alto.

Palabras clave: autoconcepto, rendimiento escolar, educación primaria, género

\section{ABSTRACT \\ School achievement and self-concept in Primary Education. Relationship and analysis by gender}




\section{RENDIMIENTO ESCOLAR Y AUTOCONCEPTO EN EDUCACIÓN PRIMARIA. RELACIÓN Y ANÁLISIS POR GÉNERO}

This paper aims, on the one hand, to analyze the relationship between self-concept and school achievement. On the other hand, to establish if there are differences in both constructs according to the gender of the students. The study included 422 primary school students, of whom 198 were boys and 224 girls. $48.3 \%$ were in fifth grade and $51.7 \%$ in sixth grade. The children studied in eight of the twelve public schools of childhood and primary education of the autonomous city of Melilla. The instruments used for data collection were two. Firstly, a register of the ratings obtained in the subjects of Environmental Knowledge, Spanish Language and Mathematics of each student, facilitated by the students' tutors. Secondly, the Test of Self-Concept Form 5 (AF-5). The results showed positive correlations between the ratings of the three subjects evaluated and three of the five dimensions of the self-concept: academic, social and family. Regarding gender analysis, they were found in the ratings for the Spanish Language subject, so that the girls scored higher than the boys. In addition, gender differences were also found in the dimensions of academic, emotional and family self-concept. Thus, girls outperformed boys on academic and family self-concept, whereas in the self-esteem the boys scored higher.

Keywords: Self-Concept, School Achievement, Primary Education, Gender

\section{INTRODUCCIÓN}

El autoconcepto es un factor altamente relevante y estudiado en la literatura científica dada su estrecha vinculación con la conducta de las personas así como con determinadas respuestas cognitivas y emocionales (Ackerman, 2003; Chamorro-Premuzic, \& Furnham, 2006; Eccles \& Wigfield, 2002). El autoconcepto constituye uno de los principales constructos en la investigación educativa y psicológica, de modo que su relación con el rendimiento o logro es un tópico relevante en la investigación académica (Huang, 2011).

Dentro del ámbito escolar, maximizar el autoconcepto académico, la autonomía así como el rendimiento escolar han sido unos de los principales logros a alcanzar (Guay, Marsh, \& Boivin, 2003; Marsh \& Craven, 2006; Valentine, DuBois, \& Cooper, 2004). Los estudios que relacionan el autoconcepto con el rendimiento escolar 0 académico son prolijos tanto en las primeras etapas educativas, como educación primaria (Möller, Retelsdorf, Köller, \& Marsh, 2011; Skaalvik, \& Skaalvik, 2013), como en educación secundaria y el ámbito universitario (Awad, 2007; Choi, 2005; Gerardi, 2005; Guay, Ratelle, Doy, \& Litalien, 2010; Thomas \& Gadbois, 2007).

Marsh y Martin (2011) Ilevaron a cabo una revisión teórica, metodológica y empírica del modelo de los efectos recíprocos (Reciprocal Effects Model-REM), el cual defiende que el autoconcepto académico y el rendimiento se refuerzan mutuamente, produciendo el uno avances en el otro, así como su extensión a otros dominios de logro como, por ejemplo, el deporte o la salud. El estudio de meta-análisis implementado por los autores permitió confirmar dichos efectos recíprocos, más allá de los posibles efectos de la autoestima u otros componentes. Además, el autoconcepto académico repercute positivamente en otros resultados educativos deseables.

Otra variable que ha sido analizada en los diferentes estudios educativos y psicológicos es el género (Chrisler \& McCreary, 2010; DiPrete \& Jennings, 2012). Así, en el estudio de meta-análisis desarrollado por Voyer y Voyer (2014), se indica que normalmente se encuentra cierta ventaja de las mujeres en el rendimiento escolar. Sus resultados ponen de manifiesto diferencias, a favor de las mujeres, para la asignatura de Lengua. También se han encontrado diferencias, por género en las investigaciones sobre el autoconcepto (Fredricks \& Eccles, 2002; Jacobs, Lanza, Osgood, Eccles, \& Wigfield, 2002; Nagy et al., 2010). Huang (2013), en un estudio de meta-análisis, identifica que las mujeres presentan un mayor autoconcepto en las materias 0 asignaturas relacionadas con el lenguaje así como con las artes en relación con los hombres. Sin embargo, los hombres exhiben un mayor autoconcepto en Matemáticas, Informática y Ciencias Sociales que las mujeres. 
En función de lo descrito, este trabajo pretende, por una parte, analizar la relación entre autoconcepto y rendimiento escolar. Por otra, establecer si existen diferencias en ambos constructos en función del género de los estudiantes.

\section{MÉTODO}

\section{Participantes}

Formaron parte de la investigación 422 alumnos de Educación Primaria, los cuales estudiaban en ocho de los doce centros públicos de educación infantil y primaria de la ciudad autónoma de Melilla (ver tabla 1). Atendiendo al género, 198 eran niños y 224 niñas. Los estudiantes, con una edad media de 10.73 años $(D T=.680)$, se hallaban cursando quinto $(48.3 \%)$ y sexto curso $(51.7 \%)$.

Tabla 1

Distribución de los participantes en función del centro de educación infantil y primaria

\begin{tabular}{lcc}
\hline Centros & Frecuencia & Porcentaje \\
\hline Centro 1 & 16 & 3.8 \\
Centro 2 & 19 & 4.5 \\
Centro 3 & 13 & 3.1 \\
Centro 4 & 126 & 29.9 \\
Centro 5 & 18 & 4.3 \\
Centro 6 & 42 & 10.0 \\
Centro 7 & 102 & 24.2 \\
Centro 8 & 86 & 20.4 \\
Total & 422 & 100.0 \\
\hline
\end{tabular}

\section{Instrumentos}

Como instrumentos de recogida de información se emplearon dos. En primer lugar, el rendimiento escolar, medido a través de las calificación obtenidas a final de curso. En segundo lugar, el Cuestionario de Autoconcepto Forma 5 (AF-5). Seguidamente se describen cada uno de ellos.

Rendimiento escolar. Las calificaciones finales obtenidas, por cada uno de los estudiantes, se obtuvieron mediante un registro, facilitado por los tutores de los alumnos, de las calificaciones en las asignaturas de Conocimiento del Medio, Lengua Castellana y Matemáticas.

Cuestionario de Autoconcepto Forma 5 (AF-5), de García y Musitu (2001). Está compuesto por 30 ítems que evalúan el autoconcepto presente en el sujeto en sus contextos Académico, Social, Emocional, Familiar y Físico. El sujeto ha de contestar según una escala atributiva de 1 a 99 según el ítem se ajuste a como el evaluado lo considere. Esnaola, Rodríguez y Goñi (2011), al analizar las propiedades psicométricas de esta prueba, indican que su fiabilidad total es de $=.74$. El índice de consistencia interna, alfa de Cronbach, calculado para el presente trabajo arrojó un valor de $=.79$. Respecto a su validez, Elosua y Muñiz (2010) confirman su estructura factorial o validez de constructo. 


\section{PROCEDIMIENTO}

\section{De recogida de información}

Por una parte, se entregó al profesorado-tutor un documento en el que se le solicitaba su colaboración en la investigación, informaba de los objetivos de la misma y explicaba cómo acceder al programa web de gestión de calificaciones del alumnado para poder descargar, en formato pdf, las calificaciones del grupo-clase. Una vez descargada esta información, se les solicitaba que apuntasen, en una tabla de doble entrada facilitada para cada estudiante, las calificaciones finales obtenidas en las asignaturas Conocimiento del Medio, Lengua Castellana y Matemáticas, esto es, Insuficiente, Suficiente, Bien, Notable o Sobresaliente.

Por otra, también a final de curso, se administró, de modo colectivo por grupo-clase, a los estudiantes de quinto y sexto curso de Educación Primaria el AF-5. Previamente, se indicaba a los alumnos que no existían respuestas buenas ni malas; que, por favor, contestasen con total sinceridad dado que se trataba de una prueba anónima, en la que no se debía escribir el nombre; que lo que iban a responder no tendría ninguna relación con las calificaciones escolares; $y$, por último, que leyeran las preguntas y, en el caso de tener alguna duda (por ejemplo, no comprender algún término), levantaran la mano y se le resolvería.

\section{De análisis estadístico de datos}

Para dar respuesta a los objetivos planteados, en primer lugar, se llevó a cabo un análisis de correlación de Pearson en el que se introdujeron como variables las calificaciones finales en las tres asignaturas de Educación Primaria descritas anteriormente así como las cinco dimensiones del autoconcepto evaluadas con el AF-5.

En segundo lugar, se realizaron dos Análisis de varianza, uno en el que como variables dependientes se introdujeron las calificaciones en las tres asignaturas registradas y otro en el que se incluyeron las diferentes dimensiones del autoconcepto. En ambos casos, la variable independiente fue el género. Para el análisis de las comparaciones post-hoc se empleó el estadístico Bonferroni.

\section{RESULTADOS}

En la tabla 2 se muestran los resultados del análisis correlacional implementado entre el autoconcepto y el rendimiento escolar. 
Tabla 2

Resultados del análisis de correlación de Pearson

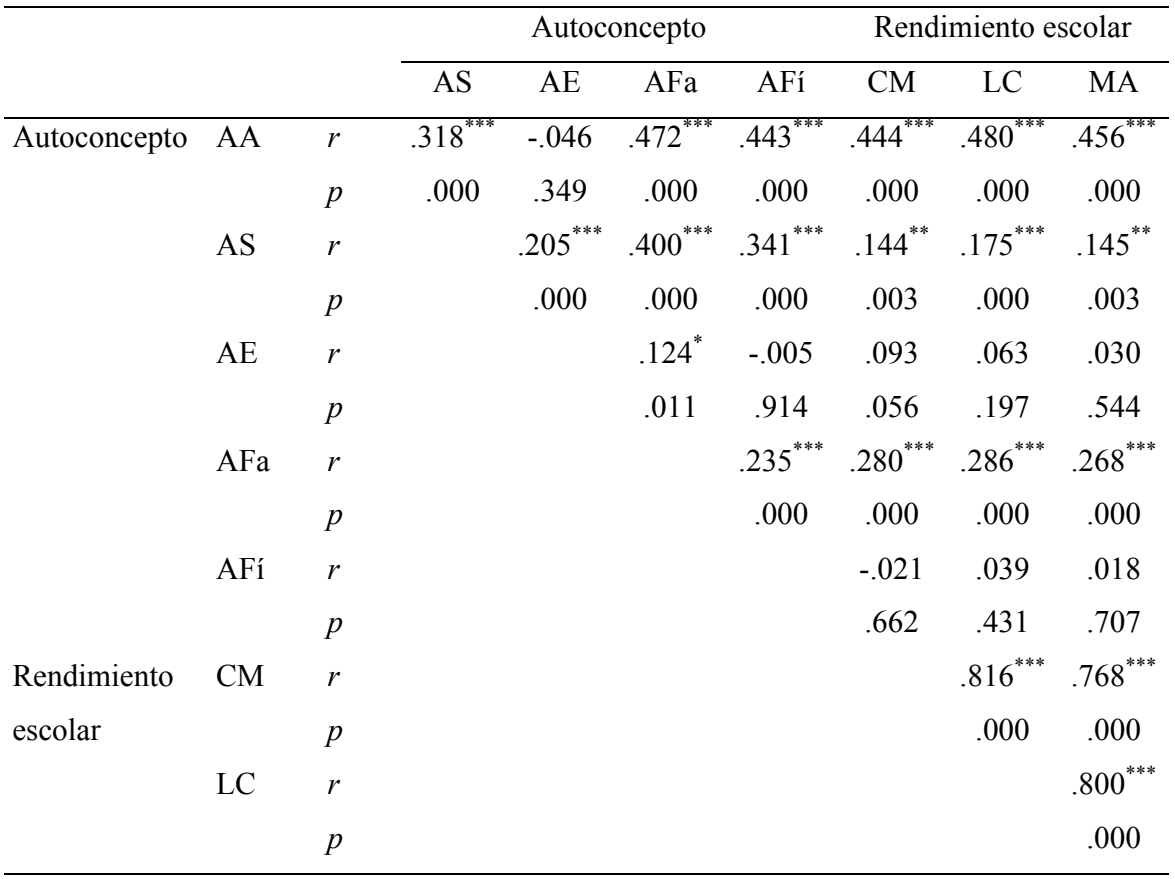

*** $p<.001, * * p<.01, * p<.05$

Nota. $\mathrm{AA}=$ Autoconcepto Académico, $\mathrm{AS}=$ Autoconcepto Social, $\mathrm{AE}=$ Autoconcepto Emocional

(Autoestima), $\mathrm{AFa}=$ Autoconcepto Familiar, AFí = Autoconcepto Físico, $\mathrm{CM}=$ Conocimiento del Medio, $\mathrm{LC}=$

Lengua Castellana, MA = Matemáticas

Se hallaron correlaciones entre las diferentes dimensiones del Autoconcepto. De este modo, el Autoconcepto Académico correlacionaba con el Autoconcepto Social, $r=.318, p=.000$; Familiar, $r$ $=.472, p=.000$; y Físico, $r=.473, p=.000$. El Autoconcepto Social, por su parte, correlacionaba positivamente con el Autoconcepto Emocional, $r=.205, p=.000$; Familiar, $r=.400, p=.000$; y Físico, $r=.341, p=.000$. El Autoconcepto Emocional se relacionaba con el Autoconcepto Familiar, $r=.124, p=.011$. Asimismo, el Autoconcepto Familiar con el Físico, $r=.235, p=.000$.

Igualmente, se identificaron altas correlaciones entre las calificaciones en Conocimiento del Medio y Lengua Castellana, $r=.816, p=.000$, además de con Mátemáticas, $r=.768, p=.000$; así como entre Lengua Castellana y Matemáticas, $r=.800, p=.000$.

En lo relativo a la posible relación entre autoconcepto y rendimiento escolar, el Autoconcepto Académico correlacionaba positivamente con las calificaciones en Conocimiento del Medio, $r=.444$, $p=.000$; Lengua Castellana, $r=.480, p=.000$; y Matemáticas, $r=.456, p=.000$. En la misma dirección, el Autoconcepto Social correlacionó con el rendimiento escolar en Conocimiento del Medio, $r$ $=.144, p=.003$; Lengua Castellana, $r=.175, p=.000$; y Matemáticas, $r=.145, p=.003$. Igualmente ocurrió para el Autoconcepto Familiar, mostrando relación con el rendimiento en las tres asignatu- 
ras, esto es, Conocimiento del Medio, $r=.280, p=.000$; Lengua Castellana, $r=.286, p=.000 ; \mathrm{y}$ Matemáticas, $r=.268, p=.000$.

Para identificar si el rendimiento escolar difería en función del género, se llevó a cabo un Anova en el que como variables dependientes se introdujeron las calificaciones finales en las tres asignaturas seleccionadas y, como variable independiente, el género. Los estadísticos descriptivos se muestran en la tabla 3.

Tabla 3

Rendimiento escolar en función del género

\begin{tabular}{lccc}
\hline Asignaturas & Género & Media & $\begin{array}{c}\text { Desviación } \\
\text { típica }\end{array}$ \\
\hline Conocimiento del Medio & Niño & 3.17 & 1.32 \\
& Niña & 3.30 & 1.36 \\
& Total & 3.24 & 1.34 \\
Lengua Castellana & Niño & 3.16 & 1.28 \\
& Niña & 3.45 & 1.21 \\
Matemáticas & Total & 3.32 & 1.25 \\
& Niño & 3.11 & 1.25 \\
& Niña & 3.25 & 1.26 \\
& Total & 3.18 & 1.26
\end{tabular}

$\overline{\text { Nota. Las calificaciones se codificaron de la siguiente forma: } 1=\text { Insuficiente, }} 2=$ Suficiente, $3=$ Bien, $4=$ Notable, $5=$ Sobresaliente

El análisis de varianza resultó significativo para la variable género, $F(1,420)=2.996, p=.031$, $E t{ }^{2}=.021$. Al respecto, en la asignatura de Lengua Castellana se encontraron diferencias en función del género, $F(1,420)=5.614, p=.018, E t a^{2}=.013$. Así, las niñas obtuvieron puntuaciones más altas que los niños, $t=.290, p=.018$.

No obstante, el análisis del rendimiento escolar en la asignatura Conocimiento del Medio, por género, no fue significativo, $F(1,420)=.929, p=.336$. Tampoco para la asignatura Matemáticas, $F(1,420)=1.278, p=.259$.

El segundo Anova desarrollado incluyó como variables dependientes las cinco dimensiones del Autoconcepto evaluadas. La puntuación media y desviación típica se muestran, en función del género, en la tabla 4. 
Tabla 4

Autoconcepto según el género

\begin{tabular}{llcc}
\hline Autoconcepto & Género & Media & $\begin{array}{c}\text { Desviación } \\
\text { típica }\end{array}$ \\
\hline Autoconcepto Académico & Niño & 7.29 & 2.22 \\
& Niña & 7.86 & 2.15 \\
& Total & 7.59 & 2.20 \\
Autoconcepto Social & Niño & 7.77 & 1.54 \\
& Niña & 7.81 & 1.50 \\
Autoconcepto Emocional & Total & 7.79 & 1.52 \\
& Niño & 6.34 & 2.11 \\
& Niña & 5.31 & 2.36 \\
Autoconcepto Familiar & Total & 5.79 & 2.30 \\
& Niño & 8.60 & 1.39 \\
& Niña & 8.88 & 1.31 \\
& Total & 8.75 & 1.35 \\
Autoconcepto Físico & Niño & 7.68 & 1.73 \\
& Niña & 7.47 & 1.91 \\
& Total & 7.57 & 1.83 \\
\hline
\end{tabular}

De forma general, se hallaron diferencias en el Autoconcepto en función del género, $F(4,417)=$ 7.608, $p=.000, E t a^{2}=.088$. Concretamente, el Autoconcepto Académico difería dependiendo del género, $F(4,417)=6.706, p=.010, E t{ }^{2}=.016$. Así, las niñas puntuaban más alto que los niños, $t$ $=.567, p=.010$. El Autoconcepto Emocional o Autoestima también era distinto según el género, $F(4$, $417)=20.534, p=.000, E_{a}{ }^{2}=.049$. Sin embargo, en este caso los niños puntuaron más alto que las niñas, $t=1.020, p=.000$. Igualmente, las diferencias fueron significativas en el Autotoconcepto Familiar, $F(4,417)=4.188, p=.041, E t a^{2}=.010$. Al igual que ocurría con el Autoconcepto Académico, las niñas alcanzaron puntuaciones más altas que los niños, $t=.277, p=.041$.

No se encontraron diferencias en el Autoconcepto Social, $F(4,417)=.062, p=.804$, ni en el Autoconcepto Físico, $F(4,417)=1.281, p=.258$.

\section{DISCUSIÓN}

En el presente trabajo se pretendía, por una parte, analizar la posible relación entre el autoconcepto y el rendimiento escolar, dada la literatura científica al respecto (Guay et al., 2010; Huang, 2011; Skaalvik, \& Skaalvik, 2013). Para ello, se ha implementado una investigación en estudiantes de quinto y sexto curso de Educación Primaria. Los resultados, en primer lugar, ponen de manifiesto correlaciones positivas entre las diferentes dimensiones del autoconcepto, evaluadas mediante el AF-5. Estos resultados coinciden con diferentes estudios sobre las características psicométricas del AF-5 (Esnaola et al., 2011; Malo, Bataller, Casas, Gras, \& González, 2011). En segundo lugar, se 
encuentran altas correlaciones entre las calificaciones o rendimiento escolar en las tres asignaturas evaluadas (Conocimiento del Medio, Lengua Castellana y Matemáticas), de modo que los estudiantes con altas calificaciones en una asignatura también obtienen en las otras dos. Este hecho puede ser debido a la implicación de diferentes factores motivacionales, entre los que se sitúa el autoconcepto 0 la autoexigencia de rendimiento elevado, como variables predictivas del rendimiento escolar (Navas, Sampascual, \& Santed, 2003). En tercer lugar, las correlaciones son positivas entre las calificaciones de las asignaturas evaluadas y tres de las cinco dimensiones del autoconcepto, esto es, académico, social y familiar. Sobre la relación entre el autoconcepto académico y el rendimiento escolar, existe una literatura prolija y cada vez más relevante (Choi, 2005; Mills, Pajares, \& Herron, 2007; Valentine et al., 2004). Así, cuando el estudiante se considera a sí mismo competente, esto es, presenta una mayor autoconfianza y altas expectativas de autoeficacia, se implica en mayor medida en su propio proceso de aprendizaje (Marsh \& Craven, 2006; Miñano \& Castejón, 2011; Rosario et al., 2009). En lo relativo a la relación entre el rendimiento escolar y el autoconcepto tanto social como familiar, no cabe duda de que, en la etapa educativa de Educación Primaria, ambos aspectos son relevantes para el ajuste académico y psicosocial. Así, la familia ocupa un papel importante desde educación infantil y, además, en los últimos cursos de Educación Primaria, tal y como se recoge en muestra de la presente investigación, la aceptación social cobra un papel privilegiado en la escala de valores de los niños (Amezcua \& Pichardo, 2004; Fernández \& Goñi, 2008; Madariaga \& Goñi, 2009). No obstante, la posición de los diferentes predictores del rendimiento escolar pueden ir variando a lo largo de la etapa de Educación Secundaria Obligatoria así como posteriormente (Esnaola, Goñi \& Madariaga, 2008; Riso, Peralbo, \& Barca, 2010).

Por otra parte, el segundo objetivo de esta investigación se centraba en identificar si existían diferencias en ambos constructos en función del género de los estudiantes. Respecto al rendimiento escolar, se han hallado diferencias a favor de las niñas en la asignatura de Lengua Castellana, lo que coincide con los resultados de otros estudios (Huang, 2013; Voyer \& Voyer, 2014).

Respecto a las diferencias por género en el autoconcepto (Fredricks \& Eccles, 2002; Jacobs et al., 2002; Nagy et al., 2010), los resultados hallados para el autoconcepto académico muestran diferencias a favor de las mujeres (Malo et al., 2011). No obstante, es necesario tener en cuenta también otros factores, como las atribuciones académicas asociadas al éxito y el fracaso escolar, dado que, por ejemplo, Inglés et al. (2012) identifican que los chicos de Educación Secundaria Obligatoria atribuyen el éxito académico a sus capacidades, mientras que las chicas lo atribuyen al esfuerzo.

También se hallan diferencias en el autoconcepto familiar, de modo que las niñas superan a los niños, lo que confirma investigaciones precedentes (García \& Gracia, 2014). Los estilos parentales así como el apoyo familiar, sus creencias y expectativas acerca de los hijos, son factores a tener en cuenta dado su influencia tanto en el desarrollo del autoconcepto como en el rendimiento académico (Álvarez et al., 2015; Olsson, 2009; Putnick et al., 2008; Ros \& Zuazagoitia, 2015).

Para finalizar, en el autoconcepto emocional o autoestima son los niños los que superan a las niñas (Inglés, Pastor, Redondo, \& García-Fernández, 2009; Garaigordobil, Durá, \& Pérez, 2005; Goñi, Fernández-Zabala, \& Infante, 2012), lo que implica un punto de reflexión y análisis para posibles programas de desarrollo de la competencia emocional de las chicas. Al respecto, los programas de alfabetización emocional, basados en el desarrollo de la inteligencia emocional, muestran claros beneficios en el desarrollo de la autoestima (Jiménez \& López-Zafra, 2009).

\section{REFERENCIAS}

Ackerman, P. L. (2003). Cognitive ability and non-ability trait determinants of expertise. Educational Researcher, 32, 15-20. doi:10.3102/0013189X032008015 
Álvarez, A., Suárez, N., Tuero, E., Núñez, J. C., Valle, A., \& Regueiro, B. (2015). Implicación familiar, autoconcepto del adolescente y rendimiento académico. European Journal of Investigation in Health, Psychology and Education, 5(3), 293-311. doi:10.1989/ejihpe.v5i3.133

Amezcua, J. A., \& Pichardo, M. C. (2004). Autoconcepto y construcción personal en la educación personal. En M. V. Trianes, \& J. A. Gallardo (Coords.), Psicología de la Educación y del Desarrollo en Contextos Escolares (pp. 181-192). Madrid: Pirámide.

Awad, G. H. (2007). The role of racial identity, academic self-concept, and self-esteem in the prediction of academic outcomes for African American students. Journal of Black Psychology, 33, 188-207. doi:10.1177/0095798407299513

Chamorro-Premuzic, T., \& Furnham, A. (2006). Intellectual competence and the intelligent personality: A third way in differential psychology. Review of General Psychology, 10, 251-267. doi:10.1037/1089-2680.10.3.251

Chrisler, J. C., \& McCreary, D.R. (2010). Handbook of Gender Research in Psychology. Volume 1: Gender Research in General and Experimental Psychology. New York, NY: Springer.

Choi, N. (2005). Self-efficacy and self-concept as predictors of college students' academic performance. Psychology in the Schools, 42, 197-205. doi:10.1002/pits.20048

DiPrete, T. A., \& Jennings, J. L. (2012). Social and behavioral skills and the gender gap in early educational achievement. Social Science Research, 41(1), 1-15. doi:10.1016/j.ssresearch.2011.09.001

Eccles, J. S., \& Wigfield, A. (2002). Motivational beliefs, values, and goals. Annual Review of Psychology, 53, 109-132. doi:10.1146/annurev.psych.53.100901.135153

Elosua, P., \& Muñiz, J. (2010). Exploring the Factorial Structure of the Self-Concept. A Sequential Approach Using CFA, MIMIC, and MACS Models, Across Gender and Two Languages. European Psychologist, 15, 58-67. doi:10.1027/1016-9040/a000006.

Esnaola, I., Goñi, A., \& Madariaga, J. M. (2008). El autoconcepto: perspectivas de investigación. Revista de Psicodidáctica, 13(1), 179-194.

Esnaola, I., Rodríguez, A., \& Goñi, E. (2011). Propiedades psicométricas del cuestionario de Autoconcepto AF5. Anales de Psicología, 27(1), 109-117. doi:10.6018/113531

Fernández, A., \& Goñi, E. (2008). El autoconcepto infantil: una revisión necesaria. INFAD. International Journal of Developmental and Educational Psychology, 1, 13-22.

Fredricks, J. A., \& Eccles, J. S. (2002). Children's competence and value beliefs from childhood through adolescence: Growth trajectories in two male-sex-typed domains. Developmental Psychology, 38(4), 519-533. doi:10.1037/0012-1649.38.4.519

Garaigordobil, M., Durá, A., \& Pérez, J. I. (2005). Síntomas psicopatológicos, problemas de conducta y autoconcepto-autoestima: Un estudio con adolescentes de 14 a 17 años. Anuario de Psicología Clínica y de la Salud, 1, 53-63.

García, F., \& Gracia, E. (2014). ¿Qué estilo de socialización parental es el idóneo en España? Un estudio con niños y adolescentes de 10 a 14 años. Infancia y Aprendizaje, 33(3), 365-384. doi:10.1174/021037010792215118

García, F., \& Musitu, G. (2001). Autoconcepto Forma 5. AF5. Madrid: TEA.

Gerardi, S. (2005). Self-concept of ability as a predictor of academic success among urban technical college students. Social Science Journal, 42, 295-300. doi:10.1016/j.soscij.2005.03.007

Goñi, E., Fernández-Zabala, A., \& Infante, F. (2012). El autoconcepto personal: diferencias asociadas a la edad y al sexo. Aula Abierta, 40(1), 39-50.

Guay, F., Marsh, H. W., \& Boivin, M. (2003). Academic self-concept and academic achievement: Developmental perspectives on their causal ordering. Journal of Educational Psychology, 95, 
124-136. doi:10.1037/0022-0663.95.1.124

Guay, F., Ratelle, C. F., Doy, A., \& Litalien, D. (2010). Academic self-concept, autonomous academic motivation, and academic achievement: Mediating and additive effects. Learning and Individual Differences, 20(6), 644-653. doi:10.1016/j.lindif.2010.08.001

Huang, C. (2011). Self-concept and academic achievement: A meta-analysis of Iongitudinal relations. Journal of School Psychology, 49(5), 505-528. doi:10.1016/j.jsp.2011.07.001

Huang, C. (2013). Gender differences in academic self-efficacy: a meta-analysis. European Journal of Psychology of Education, 28(1), 1-35. doi:10.1007/s10212-011-0097-y

Inglés, C. J., Díaz, A., García, J. M., Ruiz, C., Delgado, B., \& Martínez, M. C. (2012). Auto-atribuciones Académicas: Diferencias de Género y Curso en Estudiantes de Educación Secundaria. Revista Latinoamericana de Psicología, 44(3), 57-68. doi:10.14349/rlp.v44i3.1151

Inglés, C. J., Pastor, Y., Redondo, J., \& García-Fernández, J. M. (2009). Diferencias en función del género y el curso académico en dimensiones del autoconcepto: estudio con una muestra de adolescentes españoles. Anuario de Psicología, 40(2), 271-288.

Jacobs, J. E., Lanza, S., Osgood, D. W., Eccles, J. S., \& Wigfield, A. (2002). Changes in children's self-competence and values: Gender and domain differences across grades one through twelve. Child Development, 73(2), 509-527. doi:10.1111/1467-8624.00421

Jiménez, M. I., \& López-Zafra, E. (2009). Inteligencia emocional y rendimiento escolar: estado actual de la cuestión Revista Latinoamericana de Psicología, 41(1), 69-79.

Madariaga, J. M., \& Goñi, A. (2009). El desarrollo psicosocial. Revista de Psicodidáctica, 14, 95118.

Malo, S., Bataller, S., Casas, F., Gras, M. E., \& González, M. (2011). Análisis psicométrico de la escala multidimensional de autoconcepto AF5 en una muestra de adolescentes y adultos de Cataluña. Psicothema, 23(4), 871-878.

Marsh, H. W., \& Craven, R. G. (2006). Reciprocal effects of self-concept and performance from a multidimensional perspective: Beyond seductive pleasure and unidimensional perspectives. Perspectives on Psychological Science, 1, 133-163. doi:10.1111/j.1745-6916.2006.00010.x

Marsh, H. W., \& Martin, A. J. (2011). Academic self-concept and academic achievement: Relations and causal ordering. British Journal of Educational Psychology, 81(1), 59-77. doi:10.1348/000709910X503501

Mills, N., Pajares, F., \& Herron, C. (2007). Self-efficacy of college intermediate French students: Relation to achievement and motivation. Language Learning, 57(3), 417-442. doi:10.1111/j.1467-9922.2007.00421.x

Miñano, P., \& Castejón, J. L. (2011). Variables cognitivas y motivacionales en el rendimiento académico en Lengua y Matemáticas: un modelo estructural. Revista de Psicodidáctica, 16(2), 203230.

Möller, J., Retelsdorf, J., Köller, 0., \& Marsh, H. W. (2011). The Reciprocal Internal/External Frame of Reference Model. An Integration of Models of Relations Between Academic Achievement and Self-Concept. American Educational Research Journal, 48(6), 1315-1346. doi:10.3102/0002831211419649

Nagy, G., Watt, H., Eccles, J. S., Trautwein, U., Lüdtke, O., \& Baumert, J. (2010). The Development of Students' Mathematics Self-Concept in Relation to Gender: Different Countries, Different Trajectories. Journal or Research on Adolescence, 20(2), 482-506. doi:10.1111/j.15327795.2010.00644.x

Navas, L., Sampascual, G., \& Santed, M. A. (2003). Predicción de las calificaciones de los estudiantes. La capacidad explicativa de la inteligencia general y de la motivación. Revista de 
Psicología General y Aplicada, 56(2), 225-237.

Olsson, E. (2009). The Role of Relations: Do Disadvantaged Adolescents Benefit More from HighQuality Social Relations? Acta Sociológica, 52, 263-286.

Putnick, D. L., Bornstein, M. H., Hendricks, C., Painter, K. M., Suwalsky, J. T. D., \& Collins, W. A. (2008). Pareenting Stress, Perceived Parenting Behaviors, and Adolescent Self-Concept in European American Families. Journal of Family Psychology, 22(5), 752-762. doi:10.1037/a0013177

Riso, A., Peralbo, M., \& Barca, A. (2010). Cambios en las variables predictoras del rendimiento escolar en Enseñanza Secundaria. Psicothema, 22(4), 790-796.

Ros, I., \& Zuazagoitia, A. (2015). Implicación escolar y autoconcepto. En A. Fernández-Zabala, \& L. Revuelta (Eds.), Ajuste personal y social. Investigación psicoeducativa (pp. 119-130). Donostia: EREIN.

Rosário, P., Mourao, R., Baldaque, M., Nunes, T., Núñez, J. C., González-Pienda, J. A., ...Valle, A. (2009). Tareas para casa, autorregulación del aprendizaje y rendimiento en Matemáticas. Revista de Psicodidáctica, 14(2), 179-192.

Skaalvik, E. M., \& Skjaalvik, S. (2013). School goal structure: Associations with students' perceptions of their teachers as emotionally supportive, academic self-concept, intrinsic motivation, effort, and help seeking behavior. International Journal of Educational Research, 61, 5-14. doi:10.1016/j.ijer.2013.03.007

Thomas, C. R., \& Gadbois, S. A. (2007). Academic self-handicapping: The role of self-concept clarity and students' learning strategies. British Journal of Educational Psychology, 77, 101-119. doi:10.1348/000709905X79644

Valentine, J. C., DuBois, D. L., \& Cooper, H. (2004). The relations between self-beliefs and academic achievement: A systematic review. Educational Psychologist, 39, 111-133. doi:10.1207/s15326985ep3902_3

Voyer, D., \& Voyer, S. D. (2014). Gender differences in scholastic achievement: A meta-analysis. Psychological Bulletin, 140(4), 1174-1204. doi:10.1037/a0036620 
